\title{
On Parameter Differentiation for Integral Representations of Associated Legendre Functions ${ }^{\star}$
}

\author{
Howard S. COHL †‡ \\ $\dagger$ Applied and Computational Mathematics Division, Information Technology Laboratory, \\ National Institute of Standards and Technology, Gaithersburg, Maryland, USA \\ E-mail: hcohl@nist.gov \\ $\ddagger$ Department of Mathematics, University of Auckland, 38 Princes Str., Auckland, New Zealand \\ URL: http://www . math. auckland.ac.nz/ hcoh001/
}

Received January 19, 2011, in final form May 04, 2011; Published online May 24, 2011

doi:10.3842/SIGMA.2011.050

\begin{abstract}
For integral representations of associated Legendre functions in terms of modified Bessel functions, we establish justification for differentiation under the integral sign with respect to parameters. With this justification, derivatives for associated Legendre functions of the first and second kind with respect to the degree are evaluated at odd-halfinteger degrees, for general complex-orders, and derivatives with respect to the order are evaluated at integer-orders, for general complex-degrees. We also discuss the properties of the complex function $f: \mathbb{C} \backslash\{-1,1\} \rightarrow \mathbb{C}$ given by $f(z)=z /(\sqrt{z+1} \sqrt{z-1})$.
\end{abstract}

Key words: Legendre functions; modified Bessel functions; derivatives

2010 Mathematics Subject Classification: 31B05; 31B10; 33B10; 33B15; 33C05; 33C10

\section{Introduction}

This paper is a continuation of work which is presented in [5]. In [5], formulae were presented for derivatives of associated Legendre functions of the first kind $P_{\nu}^{\mu}$ and the second kind $Q_{\nu}^{\mu}$ with respect to their parameters, namely the degree $\nu$ and the order $\mu$, valid on the complex $z$-plane cut along the real axis from $-\infty$ to 1 (see discussion at the end of Section 3.2). The strategy applied in [5] was to differentiate integral representations of associated Legendre functions, which were given in terms of modified Bessel functions of the first and second kind, with respect to their parameters. The derivatives of the integrands, for the integral representations of associated Legendre functions given in [5], which include the derivatives with respect to the order evaluated at integer-orders for modified Bessel functions of the first and second kind, are well known (see for instance $[12, \S 3.2 .3])$.

Unfortunately, in [5], no justification for differentiation under the integral sign of the chosen integral representations of associated Legendre functions is given. In this paper, we give justification for differentiation under the integral sign for the integral representations of associated Legendre functions given in [5] and hence complete our proof for the validity of the parameter differentiation formulae given therein. The parameter differentiation formulae given in [5] are derivatives for associated Legendre functions of the first and second kind with respect to the degree, evaluated at odd-half-integer degrees, for general complex-orders, and for derivatives with respect to the order evaluated at integer-orders, for general complex-degrees.

There has been recent interest in the literature for tabulating closed-form expressions of derivatives with respect to parameters for special functions (see for instance [3]). Concerning

\footnotetext{
*This paper is a contribution to the Special Issue "Symmetry, Separation, Super-integrability and Special Functions $\left(\mathrm{S}^{4}\right)$ ". The full collection is available at http://www.emis.de/journals/SIGMA/S4.html
} 
derivatives with respect to parameters for associated Legendre functions, some formulae relating to these derivatives have been previously noted (see $[12, \S 4.4 .3]$ ), and there has been recent activity for solving open problems in this area $[3,4,5,17,18,19,20,21,22]$. For an extensive list of physical applications for derivatives with respect to parameters for associated Legendre functions with integer-order and integer-degree see [21]. For an interesting application for derivatives of associated Legendre functions evaluated at odd-half-integer degrees see [22].

This paper is organized as follows. In Section 2 we present a description of a map on a subset of the complex plane which leads to the Whipple formulae for associated Legendre functions. In Section 3 we give justification for differentiation under the integral sign for the integral representations of associated Legendre functions given in [5]. In Appendix A, we investigate properties of the complex function $z \mapsto \frac{z}{\sqrt{z^{2}-1}}$.

Throughout this paper we use the following conventions. First $\sum_{n=i}^{j} a_{n}=0$ for all $a_{1}, a_{2}, \ldots \in \mathbb{C}$, and $i, j \in \mathbb{Z}$ with $j<i$. Secondly, for any expression of the form $\left(z^{2}-1\right)^{\alpha}$, read this as

$$
\left(z^{2}-1\right)^{\alpha}:=(z+1)^{\alpha}(z-1)^{\alpha},
$$

for any fixed $\alpha \in \mathbb{C}$ and $z \in \mathbb{C} \backslash\{-1,1\}$, and principal branches are chosen (see for instance [14, $\S 4.2(\mathrm{iv})])$.

\section{The Whipple formulae for associated Legendre functions}

There is a transformation over an open subset of the complex plane which is particularly useful in studying associated Legendre functions (see [1] and [10]). This transformation, which is valid on a certain domain of the complex numbers, accomplishes the following

$$
\cosh z \leftrightarrow \operatorname{coth} w, \quad \operatorname{coth} z \leftrightarrow \cosh w, \quad \sinh z \leftrightarrow(\sinh w)^{-1}
$$

This transformation is accomplished using the map $w: \mathfrak{D} \rightarrow \mathbb{C}$, with

$$
\mathfrak{D}:=\mathbb{C} \backslash\{z \in \mathbb{C}: \operatorname{Re} z \leq 0 \text { and } \operatorname{Im} z=2 \pi n, n \in \mathbb{Z}\},
$$

and $w$ defined by

$$
w(z):=\log \operatorname{coth} \frac{z}{2} .
$$

The map $w$ is periodic with period $2 \pi i$ and is locally injective. The map $w$ restricted to $\mathfrak{D} \cap\{z \in$ $\mathbb{C}:-\pi<\operatorname{Im} z<\pi\}$ is verified to be an involution. The transformation (1) is the restriction of the mapping $w$ to this restricted domain.

This transformation is particularly useful for certain associated Legendre functions such as toroidal harmonics (see [6, 7]), associated Legendre functions of the first and second kind with odd-half-integer degree and integer-order, and for other associated Legendre functions which one might encounter in potential theory. The real argument of toroidal harmonics naturally occur in $(1, \infty)$, and these are the simultaneous ranges of both the real hyperbolic cosine and cotangent functions. One application of this map occurs with the Whipple formulae for associated Legendre functions $[8,24]$ under index (degree and order) interchange. See for instance [1, (8.2.7) and $(8.2 .8)]$, namely

$$
P_{-\mu-1 / 2}^{-\nu-1 / 2}\left(\frac{z}{\sqrt{z^{2}-1}}\right)=\sqrt{\frac{2}{\pi}} \frac{\left(z^{2}-1\right)^{1 / 4} e^{-i \mu \pi}}{\Gamma(\nu+\mu+1)} Q_{\nu}^{\mu}(z)
$$


and

$$
Q_{-\mu-1 / 2}^{-\nu-1 / 2}\left(\frac{z}{\sqrt{z^{2}-1}}\right)=-i(\pi / 2)^{1 / 2} \Gamma(-\nu-\mu)\left(z^{2}-1\right)^{1 / 4} e^{-i \nu \pi} P_{\nu}^{\mu}(z),
$$

which are valid for $\operatorname{Re} z>0$ and for all complex $\nu$ and $\mu$, except where the functions are not defined.

\section{Justification for differentiation under the integral sign}

In this section, we present and derive formulae for parameter derivatives of associated Legendre functions of the first kind $P_{\nu}^{\mu}$ and the second kind $Q_{\nu}^{\mu}$, with respect to their parameters, namely the degree $\nu$ and the order $\mu$. We cover parameter derivatives of associated Legendre functions for argument $z \in \mathbb{C} \backslash(-\infty, 1]$.

We incorporate derivatives with respect to order evaluated at integer-orders for modified Bessel functions (see $[12, \S 3.1 .3, \S 3.2 .3$, and $\S 3.3 .3]$ ) to compute derivatives with respect to the degree and the order of associated Legendre functions. Below we apply these results through certain integral representations of associated Legendre functions in terms of modified Bessel functions. Modified Bessel functions of the first and second kind respectively can be defined for all $\nu \in \mathbb{C}$ (see for instance $[23, \S 3.7]$ ) by

$$
I_{\nu}(z):=\sum_{m=0}^{\infty} \frac{(z / 2)^{\nu+2 m}}{m ! \Gamma(\nu+m+1)},
$$

and

$$
K_{\nu}(z):=\frac{\pi}{2} \frac{I_{-\nu}(z)-I_{\nu}(z)}{\sin \pi \nu} .
$$

For $\nu=n \in \mathbb{N}_{0}:=\{0,1,2, \ldots\}$, the first definition yields

$$
I_{n}(z)=I_{-n}(z) \text {. }
$$

It may be verified that

$$
K_{n}(z)=\lim _{\nu \rightarrow n} K_{\nu}(z)
$$

is well defined. The modified Bessel function of the second kind is commonly referred to as a Macdonald function.

The strategy applied in this section is to use integral representations of associated Legendre functions, expressed in terms of modified Bessel functions, and justify differentiation under the integral sign with respect to the relevant parameters.

\subsection{Parameter derivative formulas from $K_{\nu}(t)$}

It follows from $[9,(6.628 .7)]$ (see also $[15,(2.16 .6 .3)])$ that

$$
\begin{aligned}
\int_{0}^{\infty} e^{-z t} K_{\nu}(t) t^{\mu-1 / 2} d t & =\sqrt{\frac{\pi}{2}} \Gamma\left(\mu-\nu+\frac{1}{2}\right) \Gamma\left(\mu+\nu+\frac{1}{2}\right)\left(z^{2}-1\right)^{-\mu / 2} P_{\nu-1 / 2}^{-\mu}(z) \\
& =\Gamma\left(\mu-\nu+\frac{1}{2}\right)\left(z^{2}-1\right)^{-\mu / 2-1 / 4} e^{-i \pi \nu} Q_{\mu-1 / 2}^{\nu}\left(\frac{z}{\sqrt{z^{2}-1}}\right),
\end{aligned}
$$

where we used the Whipple formulae (3a), for $\operatorname{Re} z>-1$ and $\operatorname{Re} \mu>|\operatorname{Re} \nu|-1 / 2$, and the second line follows from $(3 \mathrm{~b})$ with the identity $P_{\nu}^{\mu}(z)=P_{-\nu-1}^{\mu}(z)$ (see $\left.[1,(8.2 .1)]\right)$. We would like to 
generate an analytical expression for the derivative of the associated Legendre function of the second kind with respect to its order, evaluated at integer-orders. In order to do this our strategy is to solve the above integral expression for the associated Legendre function of the second kind, differentiate with respect to the order, evaluate at integer-orders, and take advantage of the corresponding formula for differentiation with respect to the order for modified Bessel functions of the second kind (see $[12, \S 3.2 .3]$ ). Using the expression for the associated Legendre function of the second kind in (4), we solve for $Q_{\nu-1 / 2}^{\mu}(z)$ and re-express using the map in (2). This gives us the expression

$$
Q_{\nu-1 / 2}^{\mu}(z)=\frac{\left(z^{2}-1\right)^{-\nu / 2-1 / 4} e^{i \pi \mu}}{\Gamma\left(\nu-\mu+\frac{1}{2}\right)} \int_{0}^{\infty} \exp \left(\frac{-z t}{\sqrt{z^{2}-1}}\right) K_{\mu}(t) t^{\nu-1 / 2} d t .
$$

By (4) and (3b), the integral on the right-hand side of (5) is convergent for $\operatorname{Re} \frac{z}{\sqrt{z+1} \sqrt{z-1}}>-1$. By Proposition 2 in Appendix A, we have for all $z \in \mathbb{C} \backslash[-1,1]$, this is true. Hence the above integral representation (5) for the associated Legendre function of the second kind is valid for all $z \in \mathbb{C} \backslash[-1,1]$ and $\operatorname{Re} \mu>|\operatorname{Re} \nu|-1 / 2$.

In order to justify differentiation under the integral sign we use the following well-known corollary of the bounded convergence theorem (cf. [11, $\S 8.2]$ ).

Proposition 1. Let $(X, \mu)$ be a measure space, $U \subset \mathbb{R}$ open and $f: X \times U \rightarrow \mathbb{R}$ a function. Suppose

1) for all $y \in U$ the function $x \mapsto f(x, y)$ is measurable,

2) $\frac{\partial f}{\partial y}(x, y)$ exists for all $(x, y) \in X \times U$,

3) there exists $g \in \mathcal{L}^{1}(X)$ such that $\left|\frac{\partial f}{\partial y}(x, y)\right| \leq g(x)$ for all $(x, y) \in X \times U$.

Then the function $y \mapsto \int_{X} f(x, y) d \mu(x)$ is differentiable on $U$ and

$$
\frac{d}{d y}\left(\int_{X} f(x, y) d \mu(x)\right)=\int_{X} \frac{\partial f}{\partial y}(x, y) d \mu(x) .
$$

We call $g$ a $\mathcal{L}^{1}$-majorant.

We wish to differentiate (5) with respect to the order $\mu$ and evaluate at $\mu_{0}= \pm m$, where $m \in \mathbb{N}_{0}$. The derivative of the modified Bessel function of the second kind with respect to its order (see $[12, \S 3.2 .3]$ ) is given by

$$
\left[\frac{\partial}{\partial \mu} K_{\mu}(t)\right]_{\mu= \pm m}= \pm m ! \sum_{k=0}^{m-1} \frac{2^{m-1-k}}{k !(m-k)} t^{k-m} K_{k}(t)
$$

(see for instance $[3,(1.14 .2 .2)]$ ). For a fixed $t, K_{\mu}(t)$ is an even function of $\mu \in \mathbb{R}$ (see [14, $(10.27 .3)])$, i.e.

$$
K_{-\mu}(t)=K_{\mu}(t)
$$

and for $\mu \in[0, \infty), K_{\mu}(t)$ is a strictly increasing function of $\mu$. For a fixed $t, \partial K_{\mu}(t) / \partial \mu$ is an odd function of $\mu \in \mathbb{R}$ and for $\mu \in[0, \infty)$, and $\partial K_{\mu}(t) / \partial \mu$ is also a strictly increasing function of $\mu$. Using (6) we can make the following estimate

$$
\left|\frac{\partial}{\partial \mu} K_{\mu}(t)\right|<\left.\frac{\partial K_{\tau}(t)}{\partial \tau}\right|_{\tau= \pm(m+1)},
$$

for all $\mu \in\left(\mu_{0}-1, \mu_{0}+1\right)$. 
To justify differentiation under the integral sign in (5), with respect to $\mu$, evaluated at $\mu_{0}$, we use Proposition 1. If we fix $z$ and $\nu$, the integrand of (5) can be given by the function $f: \mathbb{R} \times(0, \infty) \rightarrow \mathbb{C}$ defined by

$$
f(\mu, t):=\exp \left(\frac{-z t}{\sqrt{z^{2}-1}}\right) t^{\nu-1 / 2} K_{\mu}(t)
$$

Since $\partial K_{\mu}(t) / \partial \mu$ is a strictly increasing function of $\mu \in[0, \infty)$, we have for all $\mu \in\left(\mu_{0}-1, \mu_{0}+1\right)$

$$
\begin{aligned}
\left|\frac{\partial f}{\partial \mu}(\mu, t)\right| & =\exp \left[\operatorname{Re}\left(\frac{-z t}{\sqrt{z^{2}-1}}\right)\right] t^{\operatorname{Re} \nu-1 / 2}\left|\frac{\partial}{\partial \mu} K_{\mu}(t)\right| \\
& <\exp \left[\operatorname{Re}\left(\frac{-z t}{\sqrt{z^{2}-1}}\right)\right] t^{\operatorname{Re} \nu-1 / 2}\left|\left[\frac{\partial}{\partial \tau} K_{\tau}(t)\right]_{\tau= \pm(m+1)}\right| \\
& =\exp \left[\operatorname{Re}\left(\frac{-z t}{\sqrt{z^{2}-1}}\right)\right] t^{\operatorname{Re} \nu-1 / 2}\left|\left[\frac{\partial}{\partial \tau} K_{\tau}(t)\right]_{\tau=m+1}\right| \\
& \leq \exp \left[\operatorname{Re}\left(\frac{-z t}{\sqrt{z^{2}-1}}\right)\right] t^{\operatorname{Re} \nu-1 / 2}(m+1) ! \sum_{k=0}^{m} \frac{2^{m-k}}{k !(m+1-k)} t^{k-m-1} K_{k}(t) \\
& \leq \exp \left[\operatorname{Re}\left(\frac{-z t}{\sqrt{z^{2}-1}}\right)\right] t^{\operatorname{Re} \nu-1 / 2}(m+1) ! 2^{m} t^{-1} K_{m}(t)=: g(t),
\end{aligned}
$$

where we used (6) and the fact that $K_{k}(t) \leq K_{m}(t)$ for all $k \in\{0, \ldots, m-1\}$. Then $g$ is a $\mathcal{L}^{1}$ majorant for the derivative of the integrand, since the integral (5) converges for $\operatorname{Re}\left(z / \sqrt{z^{2}-1}\right)>$ -1 and $\operatorname{Re} \nu>m-1 / 2$.

The conditions for differentiating under the integral sign have been satisfied and we can re-write (5) as

$$
\begin{aligned}
{\left[\frac{\partial}{\partial \mu} Q_{\nu-1 / 2}^{\mu}(z)\right]_{\mu= \pm m}=} & \left(z^{2}-1\right)^{-\nu / 2-1 / 4}\left[\frac{\partial}{\partial \mu} \frac{e^{i \pi \mu}}{\Gamma\left(\nu-\mu+\frac{1}{2}\right)}\right]_{\mu= \pm m} \\
& \times \int_{0}^{\infty} \exp \left(\frac{-z t}{\sqrt{z^{2}-1}}\right) K_{ \pm m}(t) t^{\nu-1 / 2} d t \\
& +\frac{\left(z^{2}-1\right)^{-\nu / 2-1 / 4}(-1)^{m}}{\Gamma\left(\nu \mp m+\frac{1}{2}\right)} \\
& \times \int_{0}^{\infty} \exp \left(\frac{-z t}{\sqrt{z^{2}-1}}\right) t^{\nu-1 / 2}\left[\frac{\partial}{\partial \mu} K_{\mu}(t)\right]_{\mu= \pm m} d t .
\end{aligned}
$$

The derivative from the first term is given as

$$
\left[\frac{\partial}{\partial \mu} \frac{e^{i \pi \mu}}{\Gamma\left(\nu-\mu+\frac{1}{2}\right)}\right]_{\mu= \pm m}=\frac{(-1)^{m}}{\Gamma\left(\nu \mp m+\frac{1}{2}\right)}\left[i \pi+\psi\left(\nu \mp m+\frac{1}{2}\right)\right]
$$

where the $\psi$ is the digamma function defined in terms of the derivative of the gamma function,

$$
\frac{d}{d z} \Gamma(z):=\psi(z) \Gamma(z)
$$

for $z \in \mathbb{C} \backslash\left(-\mathbb{N}_{0}\right)$.

Substituting these expressions for the derivatives into the two integrals and using the map in (2) to re-evaluate these integrals in terms of associated Legendre functions gives the following 
general expression for the derivative of the associated Legendre function of the second kind with respect to its order evaluated at integer-orders as

$$
\begin{aligned}
\frac{\Gamma\left(\nu \mp m+\frac{1}{2}\right)}{\Gamma\left(\nu-m+\frac{1}{2}\right)}\left[\frac{\partial}{\partial \mu} Q_{\nu-1 / 2}^{\mu}(z)\right]_{\mu= \pm m}= & {\left[i \pi+\psi\left(\nu \mp m+\frac{1}{2}\right)\right] Q_{\nu-1 / 2}^{m}(z) } \\
& \pm m ! \sum_{k=0}^{m-1} \frac{(-1)^{k-m}\left(z^{2}-1\right)^{(k-m) / 2}}{k !(m-k) 2^{k-m+1}} Q_{\nu+k-m-1 / 2}^{k}(z) .
\end{aligned}
$$

If we start with the expression for the associated Legendre function of the first kind in (4) and solve for $P_{\nu-1 / 2}^{-\mu}(z)$ we have

$$
P_{\nu-1 / 2}^{-\mu}(z)=\sqrt{\frac{2}{\pi}} \frac{\left(z^{2}-1\right)^{\mu / 2}}{\Gamma\left(\mu-\nu+\frac{1}{2}\right) \Gamma\left(\mu+\nu+\frac{1}{2}\right)} \int_{0}^{\infty} e^{-z t} K_{\nu}(t) t^{\mu-1 / 2} d t .
$$

To justify differentiation under the integral sign in (8), with respect to $\nu$, evaluated at $\nu= \pm n$, where $n \in \mathbb{N}_{0}$, we use as similar argument as in (5) only with modification $\mu \mapsto \nu$ and $m \mapsto n$. The same modified $\mathcal{L}^{1}$-majorant will work for the derivative of this integrand, since the integral (8) converges for $\operatorname{Re} z>-1$ and $\operatorname{Re} \nu>|\operatorname{Re} \mu|-1 / 2$.

The conditions for differentiating under the integral sign have been satisfied and we can re-write (8) as

$$
\begin{aligned}
{\left[\frac{\partial}{\partial \nu} P_{\nu-1 / 2}^{-\mu}(z)\right]_{\nu= \pm n}=} & \sqrt{\frac{2}{\pi}}\left(z^{2}-1\right)^{\mu / 2}\left[\frac{\partial}{\partial \nu} \frac{1}{\Gamma\left(\mu-\nu+\frac{1}{2}\right) \Gamma\left(\mu+\nu+\frac{1}{2}\right)}\right]_{\nu= \pm n} \\
& \times \int_{0}^{\infty} e^{-z t} K_{ \pm n}(t) t^{\mu-1 / 2} d t+\sqrt{\frac{2}{\pi}} \frac{\left(z^{2}-1\right)^{\mu / 2}}{\Gamma\left(\mu \mp n+\frac{1}{2}\right) \Gamma\left(\mu \pm n+\frac{1}{2}\right)} \\
& \times \int_{0}^{\infty} e^{-z t} t^{\mu-1 / 2}\left[\frac{\partial}{\partial \nu} K_{\nu}(t)\right]_{\nu= \pm n} d t .
\end{aligned}
$$

The derivative from the first term in (9) is given as

$$
\left[\frac{\partial}{\partial \nu} \frac{1}{\Gamma\left(\mu-\nu+\frac{1}{2}\right) \Gamma\left(\mu+\nu+\frac{1}{2}\right)}\right]_{\nu= \pm n}=\frac{\psi\left(\mu \mp n+\frac{1}{2}\right)-\psi\left(\mu \pm n+\frac{1}{2}\right)}{\Gamma\left(\mu \pm n+\frac{1}{2}\right) \Gamma\left(\mu \mp n+\frac{1}{2}\right)} .
$$

Substituting this expression for the derivative and that given in (6) yields the following general expression for the derivative of the associated Legendre function of the first kind with respect to its degree evaluated at odd-half-integer degrees as

$$
\begin{gathered}
\pm\left[\frac{\partial}{\partial \nu} P_{\nu-1 / 2}^{-\mu}(z)\right]_{\nu= \pm n}=\left[\psi\left(\mu-n+\frac{1}{2}\right)-\psi\left(\mu+n+\frac{1}{2}\right)\right] P_{n-1 / 2}^{-\mu}(z) \\
\quad+\frac{n !}{\Gamma\left(\mu+n+\frac{1}{2}\right)} \sum_{k=0}^{n-1} \frac{\Gamma\left(\mu-n+2 k+\frac{1}{2}\right)\left(z^{2}-1\right)^{(n-k) / 2}}{k !(n-k) 2^{k-n+1}} P_{k-1 / 2}^{-\mu+n-k}(z) .
\end{gathered}
$$

If one makes a global replacement $-\mu \mapsto \mu$, using the properties of gamma and digamma functions, this result reduces to

$$
\begin{aligned}
& \pm\left[\frac{\partial}{\partial \nu} P_{\nu-1 / 2}^{\mu}(z)\right]_{\nu= \pm n}=\left[\psi\left(\mu+n+\frac{1}{2}\right)-\psi\left(\mu-n+\frac{1}{2}\right)\right] P_{n-1 / 2}^{\mu}(z) \\
& \quad+n ! \Gamma\left(\mu-n+\frac{1}{2}\right) \sum_{k=0}^{n-1} \frac{\left(z^{2}-1\right)^{(n-k) / 2}}{\Gamma\left(\mu+n-2 k+\frac{1}{2}\right) k !(n-k) 2^{k-n+1}} P_{k-1 / 2}^{\mu+n-k}(z) .
\end{aligned}
$$




\subsection{Parameter derivative formulas from $I_{\nu}(t)$}

Starting this time with $[9,(6.624 .5)]$ (see also $[15,(2.15 .3 .2)]$ ), we have for $\operatorname{Re} z>1$ and $\operatorname{Re} \mu>$ $-\operatorname{Re} \nu-1 / 2$,

$$
\begin{aligned}
\int_{0}^{\infty} e^{-z t} I_{\nu}(t) t^{\mu-1 / 2} d t & =\sqrt{\frac{2}{\pi}} e^{-i \pi \mu}\left(z^{2}-1\right)^{-\mu / 2} Q_{\nu-1 / 2}^{\mu}(z) \\
& =\Gamma\left(\mu+\nu+\frac{1}{2}\right)\left(z^{2}-1\right)^{-\mu / 2-1 / 4} P_{\mu-1 / 2}^{-\nu}\left(\frac{z}{\sqrt{z^{2}-1}}\right),
\end{aligned}
$$

where we used again the Whipple formulae (3a).

We will use this particular integral representation of associated Legendre functions to compute certain derivatives of the associated Legendre functions with respect to the degree and the order. We start with the integral representation of the associated Legendre function of the second kind (11), namely

$$
Q_{\nu-1 / 2}^{\mu}(z)=\sqrt{\frac{\pi}{2}} e^{i \pi \mu}\left(z^{2}-1\right)^{\mu / 2} \int_{0}^{\infty} e^{-z t} t^{\mu-1 / 2} I_{\nu}(t) d t
$$

To justify differentiation under the integral sign in (12), with respect to $\nu$, evaluated at $\nu_{0}= \pm n$, where $n \in \mathbb{N}:=\{1,2,3, \ldots\}$, we use again Proposition 1 . If we fix $z$ and $\mu$, the integrand of (12) can be given by the function $f: \mathbb{R} \times(0, \infty) \rightarrow \mathbb{C}$ defined by

$$
f(\nu, t):=e^{-z t} t^{\mu-1 / 2} I_{\nu}(t)
$$

We use the following integral representation for the derivative with respect to the order of the modified Bessel function of the first kind (see [2, (75)])

$$
\frac{\partial I_{\nu}(t)}{\partial \nu}=-\nu \int_{0}^{t} K_{0}(t-x) I_{\nu}(x) x^{-1} d x
$$

Let $\delta \in(0,1)$ and $M>2$. Consider $g:(0, \infty) \rightarrow[0, \infty)$ defined by

$$
g(t):=M e^{-t \operatorname{Re} z} t^{\operatorname{Re} \mu-1 / 2} \int_{0}^{t} K_{0}(t-x) I_{\delta}(x) x^{-1} d x .
$$

Using (13) we have for all $\nu \in(\delta, M)$

$$
\begin{aligned}
\left|\frac{\partial f(\nu, t)}{\partial \nu}\right| & =e^{-t \operatorname{Re} z} t^{\operatorname{Re} \mu-1 / 2}\left|\frac{\partial I_{\nu}(t)}{\partial \nu}\right|=\nu e^{-t \operatorname{Re} z} t^{\operatorname{Re} \mu-1 / 2} \int_{0}^{t} K_{0}(t-x) I_{\nu}(x) x^{-1} d x \\
& \leq M e^{-t \operatorname{Re} z} t^{\operatorname{Re} \mu-1 / 2} \int_{0}^{t} K_{0}(t-x) I_{\delta}(x) x^{-1} d x=g(t),
\end{aligned}
$$

since for fixed $t, \nu \mapsto I_{\nu}(t)$ is strictly decreasing. Now we show that $g \in \mathcal{L}^{1}$. The integral of $g$ over its domain is

$$
\int_{0}^{\infty} g(t) d t=M \int_{0}^{\infty} e^{-t \operatorname{Re} z} t^{\operatorname{Re} \mu-1 / 2} \int_{0}^{t} K_{0}(t-x) I_{\delta}(x) x^{-1} d x d t .
$$

Making a change of variables in the integral, $(x, t) \mapsto(x, y)$ with $y=t-x$, yields

$$
\int_{0}^{\infty} g(t) d t=M \int_{0}^{\infty} e^{-y \operatorname{Re} z} K_{0}(y) \int_{0}^{\infty} e^{-x \operatorname{Re} z}(x+y)^{\operatorname{Re} \mu-1 / 2} x^{-1} I_{\delta}(x) d x d y .
$$


First we show that $g$ is integrable in a neighborhood of zero. Suppose $\operatorname{Re} \mu-1 / 2<0, x, y \in(0,1]$ and $a \in(0,1)$. Then

$$
(x+y)^{\operatorname{Re} \mu-1 / 2}=(x+y)^{-a}(x+y)^{\operatorname{Re} \mu-1 / 2+a} \leq y^{-a} \max \left(2^{\operatorname{Re} \mu-1 / 2+a}, x^{\operatorname{Re} \mu-1 / 2+a}\right) .
$$

Since $K_{0}(y) \sim-\log (y)[14,(10.30 .3)]$ it follows that

$$
\int_{0}^{1} K_{0}(y) y^{-a} d y<\infty
$$

Furthermore since $I_{\delta}(x) \sim(x / 2)^{\delta} / \Gamma(\delta+1)[14,(10.30 .1)]$ it follows that

$$
\int_{0}^{1} I_{\delta}(x) x^{-1} d x<\infty .
$$

Now we show that

$$
\int_{0}^{1} I_{\delta}(x) x^{\operatorname{Re} \mu-1 / 2+a-1} d x
$$

is convergent if $\operatorname{Re} \mu-1 / 2+a+\delta>0$. If we define

$$
\epsilon:=\frac{\operatorname{Re} \mu+\nu_{0}+\frac{1}{2}}{3}>0,
$$

then $\operatorname{Re} \mu=-\nu_{0}-1 / 2+3 \epsilon$. Therefore if we take $a:=1-\epsilon$ and $\delta:=\nu_{0}-\epsilon<\nu_{0}$ then

$$
\operatorname{Re} \mu-\frac{1}{2}+a+\delta=\epsilon>0
$$

and hence (14) is convergent and thus $g$ is integrable near the origin. If $\operatorname{Re} \mu-1 / 2 \geq 0$ then similarly $g$ is integrable near the origin.

Now we show that $g$ is integrable. Suppose $\operatorname{Re} \mu-1 / 2>0$. Then

$$
\left.(x+y)^{\operatorname{Re} \mu-1 / 2} \leq[2 \max (x, y))\right]^{\operatorname{Re} \mu-1 / 2}=2^{\operatorname{Re} \mu-1 / 2} \max \left(x^{\operatorname{Re} \mu-1 / 2}, y^{\operatorname{Re} \mu-1 / 2}\right)
$$

for all $x, y \geq 0$. For $y \rightarrow \infty$ one has $K_{\nu}(y) \sim \sqrt{\pi /(2 y)} e^{-y}[13$, p. 250]. Hence it follows that

$$
\int_{1}^{\infty} K_{0}(y) e^{-y \operatorname{Re} z} y^{\operatorname{Re} \mu-1 / 2} d y<\infty
$$

and

$$
\int_{1}^{\infty} K_{0}(y) e^{-y \operatorname{Re} z} d y<\infty
$$

Furthermore since for $x \rightarrow \infty, I_{\delta}(x) \sim e^{x} / \sqrt{2 \pi x}$ [13, p. 251] it follows that

$$
\int_{1}^{\infty} e^{-x \operatorname{Re} z} I_{\delta}(x) x^{\operatorname{Re} \mu-3 / 2} d x<\infty
$$

and

$$
\int_{1}^{\infty} e^{-x \operatorname{Re} z} I_{\delta}(x) x^{-1} d x<\infty
$$

If $\operatorname{Re} \mu-1 / 2 \leq 0$ then similarly $g$ is integrable. 
Therefore $g$ is a $\mathcal{L}^{1}$-majorant for the derivative with respect to $\nu$ of the integrand in (12). It is unclear whether differentiation under the integral sign is also possible for $\nu_{0}=0$. However, we show below that our derived results for derivatives with respect to the degree for associated Legendre functions match up with the to be derived results for degree $\nu=0$. Relatively little is known about the properties of Bessel functions in relation to operations (differentiation and integration) with respect to their order (cf. [2]).

Differentiating with respect to the degree $\nu$ and evaluating at $\nu= \pm n$, where $n \in \mathbb{N}$, one obtains

$$
\left[\frac{\partial}{\partial \nu} Q_{\nu-1 / 2}^{\mu}(z)\right]_{\nu= \pm n}=\sqrt{\frac{\pi}{2}} e^{i \pi \mu}\left(z^{2}-1\right)^{\mu / 2} \int_{0}^{\infty} e^{-z t} t^{\mu-1 / 2}\left[\frac{\partial}{\partial \nu} I_{\nu}(t)\right]_{\nu= \pm n} d t .
$$

The derivative of the modified Bessel function of the first kind with respect to the order evaluated at integer-orders $(15)$ (see $[12, \S 3.2 .3]$ ) is given by

$$
\left[\frac{\partial}{\partial \nu} I_{\nu}(t)\right]_{\nu= \pm n}=(-1)^{n+1} K_{n}(t) \pm n ! \sum_{k=0}^{n-1} \frac{(-1)^{k-n}}{k !(n-k)} \frac{t^{k-n}}{2^{k-n+1}} I_{k}(t)
$$

(see for instance [3, (1.13.2.1)]).

Inserting (16) into (15) and using (4) and (11), we obtain the following general expression for the derivative of the associated Legendre function of the second kind with respect to its degree evaluated at odd-half-integer degrees as

$$
\begin{aligned}
{\left[\frac{\partial}{\partial \nu} Q_{\nu-1 / 2}^{\mu}(z)\right]_{\nu= \pm n}=} & -\sqrt{\frac{\pi}{2}} e^{i \pi \mu} \Gamma\left(\mu-n+\frac{1}{2}\right)\left(z^{2}-1\right)^{-1 / 4} Q_{\mu-1 / 2}^{n}\left(\frac{z}{\sqrt{z^{2}-1}}\right) \\
& \pm n ! \sum_{k=0}^{n-1} \frac{\left(z^{2}-1\right)^{(n-k) / 2}}{2^{k-n+1} k !(n-k)} Q_{k-1 / 2}^{\mu+k-n}(z) .
\end{aligned}
$$

Note that

$$
\left[\frac{\partial}{\partial \nu} Q_{\nu-1 / 2}^{\mu}(z)\right]_{\nu=0}=-\sqrt{\frac{\pi}{2}} e^{i \pi \mu} \Gamma\left(\mu+\frac{1}{2}\right)\left(z^{2}-1\right)^{-1 / 4} Q_{\mu-1 / 2}\left(\frac{z}{\sqrt{z^{2}-1}}\right),
$$

by [12]. Therefore (17) is also valid if $\nu=0$.

Finally, we obtain a formula for the derivative with respect to the order for the associated Legendre function of the first kind evaluated at integer-orders. In order to do this we use the integral expression for the associated Legendre function of the first kind given by (11) and the map given in (2) to convert to the appropriate argument. Now use the negative-order condition for associated Legendre functions of the first kind (see for example [8, (22)]) to convert to a positive order, namely

$$
\begin{aligned}
P_{\nu-1 / 2}^{\mu}(z)= & \frac{2}{\pi} e^{-i \mu \pi} \sin (\mu \pi) Q_{\nu-1 / 2}^{\mu}(z) \\
& +\frac{\left(z^{2}-1\right)^{-\nu / 2-1 / 4}}{\Gamma\left(\nu-\mu+\frac{1}{2}\right)} \int_{0}^{\infty} \exp \left(\frac{-z t}{\sqrt{z^{2}-1}}\right) I_{\mu}(t) t^{\nu-1 / 2} d t
\end{aligned}
$$

By (11) and (3b), the integral on the right-hand side of (18) converges for $\operatorname{Re} \frac{z}{\sqrt{z+1} \sqrt{z-1}}>1$. By Proposition 2 in Appendix A, we only have $\operatorname{Re} \frac{z}{\sqrt{z+1} \sqrt{z-1}}>0$ for $z \in \mathbb{C} \backslash[-1,1]$. Therefore the above integral representation (18) for the associated Legendre function of the first kind will not be valid for the full region $z \in \mathbb{C} \backslash[-1,1]$, but only for a doubly-connected open subset of $\mathbb{C}$ which is symmetric about the real and imaginary axes which includes the real segment 
$z \in(1, \infty)$ and has boundary in quadrant I, given by the curve $\operatorname{Re} \frac{z}{\sqrt{z+1} \sqrt{z-1}}=1$. For a detailed discussion of this curve, see the end of Appendix A.

To justify differentiation under the integral sign in (18), with respect to $\mu$, evaluated at $\mu= \pm m$, where $m \in \mathbb{N}$, we use as similar argument as in (12), only with modification $\nu \mapsto \mu$ and $n \mapsto m$. The same modified $\mathcal{L}^{1}$-majorant will work for the derivative of this integrand, since the integral (18) converges for $\operatorname{Re}\left(z / \sqrt{z^{2}-1}\right)>1$ and $\operatorname{Re} \mu>-\operatorname{Re} \nu-1 / 2$. Since we were unable to justify differentiation under the integral for $\nu=0$ before, the case for differentiation under the integral (18) with respect to $\mu$ evaluated at $\mu=0$ remains open. However, below we show that our derived results for derivatives with respect to the order for associated Legendre functions match up to previously established results in the literature for order $\mu=0$.

Differentiating both sides of the resulting expression with respect to the order $\mu$ and evaluating at $\mu= \pm m$, where $m \in \mathbb{N}$ yields

$$
\begin{aligned}
& {\left[\frac{\partial}{\partial \mu} P_{\nu-1 / 2}^{\mu}(z)\right]_{\mu= \pm m}=2 Q_{\nu-1 / 2}^{ \pm m}(z)+\left(z^{2}-1\right)^{-\nu / 2-1 / 4}} \\
& \quad \times\left\{\frac{\partial}{\partial \mu}\left[\Gamma\left(\nu-\mu+\frac{1}{2}\right)\right]^{-1}\right\}_{\mu= \pm m} \int_{0}^{\infty} \exp \left(\frac{-z t}{\sqrt{z^{2}-1}}\right) I_{ \pm m}(t) t^{\nu-1 / 2} d t \\
& \quad+\frac{\left(z^{2}-1\right)^{-\nu / 2-1 / 4}}{\Gamma\left(\nu \mp m+\frac{1}{2}\right)} \int_{0}^{\infty} \exp \left(\frac{-z t}{\sqrt{z^{2}-1}}\right) t^{\nu-1 / 2}\left[\frac{\partial}{\partial \mu} I_{\mu}(t)\right]_{\mu= \pm m} d t
\end{aligned}
$$

The derivative of the reciprocal of the gamma function reduces to

$$
\left\{\frac{\partial}{\partial \mu}\left[\Gamma\left(\nu-\mu+\frac{1}{2}\right)\right]^{-1}\right\}_{\mu= \pm m}=\frac{\psi\left(\nu \mp m+\frac{1}{2}\right)}{\Gamma\left(\nu \mp m+\frac{1}{2}\right)} .
$$

The derivative with respect to order for the modified Bessel function of the first kind is given in (16). The integrals are easily obtained by applying the map given by (2) as necessary to (4) and (11). Hence by also using standard properties of associated Legendre, gamma, and digamma functions we obtain the following compact form

$$
\begin{aligned}
\frac{\Gamma\left(\nu \mp m+\frac{1}{2}\right)}{\Gamma\left(\nu-m+\frac{1}{2}\right)}\left[\frac{\partial}{\partial \mu} P_{\nu-1 / 2}^{\mu}(z)\right]_{\mu= \pm m}= & Q_{\nu-1 / 2}^{m}(z)+\psi\left(\nu \mp m+\frac{1}{2}\right) P_{\nu-1 / 2}^{m}(z) \\
& \pm m ! \sum_{k=0}^{m-1} \frac{(-1)^{k-m}\left(z^{2}-1\right)^{(k-m) / 2}}{2^{k-m+1} k !(m-k)} P_{\nu+k-m-1 / 2}^{k}(z) .
\end{aligned}
$$

Note that

$$
\left[\frac{\partial}{\partial \mu} P_{\nu-1 / 2}^{\mu}(z)\right]_{\mu=0}=Q_{\nu-1 / 2}(z)+\psi\left(\nu+\frac{1}{2}\right) P_{\nu-1 / 2}(z),
$$

by $[12, \S 4.4 .3]$. So (19) is also valid if $\mu=0$.

The integral representations (5), (8), (12), and (18), which are used to obtain the parameter derivative formulae for associated Legendre functions of the first and second kind presented in this paper (7), (10), (17), and (19), are each convergent, in terms of the argument $z$, in their own specific regions of the complex plane. The presented parameter derivative formulae are given in terms of finite sums over associated Legendre functions which are analytic functions on the domain $\mathbb{C} \backslash(-\infty, 1]$. Therefore, these formulae provide an analytic continuation for the parameter derivatives to the domain given by the cut plane with argument $z \in \mathbb{C} \backslash(-\infty, 1]$. 


\section{A Properties of the function $z \mapsto z / \sqrt{z^{2}-1}$}

In this paper we make use of integral representations of associated Legendre functions, namely (4) and (11), and frequently take advantage of the Whipple formulae (3a) and (3b). The Whipple formulae directly relate Legendre functions of the first and second kind evaluated at arguments $z$ and $z / \sqrt{z^{2}-1}$ respectively. Hence it is useful in conjunction with the Whipple formulae, to understand the mapping properties of $z \mapsto z / \sqrt{z^{2}-1}$. In particular one would like to know the behavior of the real part of this function in regard to domains of convergence for the integral representations of associated Legendre functions which are used.

Proposition 2. Define the function $f: \mathbb{C} \backslash\{-1,1\} \rightarrow \mathbb{C}$ by

$$
f(z)=\frac{z}{\sqrt{z^{2}-1}}:=\frac{z}{\sqrt{z+1} \sqrt{z-1}}
$$

where the principal branch of the square roots are chosen. This function $f$ has the following properties.

1. $\left.f\right|_{\mathbb{C} \backslash[-1,1]}$ is even and $\left.f\right|_{(-1,1) \pm i 0}$ is odd, where $\pm i 0:=i \lim _{x \rightarrow 0^{ \pm}} x$.

2. The sets $(0,1) \pm i 0$ and $(-1,0) \pm i 0$ are mapped onto $i\left\{\begin{array}{c}(-\infty, 0) \\ (0, \infty)\end{array}\right\}$ and $i\left\{\begin{array}{c}(0, \infty) \\ (-\infty, 0)\end{array}\right\}$ respectively, where $\pm i \infty:=i \lim _{x \rightarrow \pm \infty} x$.

3. The sets $i(-\infty, 0)$ and $i(0, \infty)$ are both mapped to $(0,1)$.

4. $f(0 \pm i 0)=0$.

5. If $z \in \mathbb{C} \backslash[-1,1]$ then $\operatorname{Re} f(z)>0$.

Proof. When $z \neq 0$ and the exponent $w$ is any complex number, then $z^{w}$ is defined by the equation

$$
z^{w}:=\exp (w \log z)
$$

where the exponential function can be defined over the entire complex plane using the power series definition

$$
\exp (z):=\sum_{n=0}^{\infty} \frac{z^{n}}{n !},
$$

and the logarithmic function is defined for points $z=r e^{i \arg z}$, with $r>0$, as

$$
\log z:=\log r+i \arg z
$$

Recall that if $z \in \mathbb{C} \backslash\{0\}$, then $\arg z$ (often referred to as the argument, amplitude or phase) is given by the angle measured from the positive real axis to the vector representing $z$. The angle is positive if measured anticlockwise and we choose the $\arg z \in(-\pi, \pi)$. The principal branch of the square root $\sqrt{z}$ (with branch cut along $(-\infty, 0]$ ) is given by that unique branch of the square root which is non-negative for $z \in(0, \infty)$. Using this branch of the square root, the product $\sqrt{z+1} \sqrt{z-1}$ is well-defined and continuous in $z \in(-\infty,-1)$. A branch cut along $[-1,1]$ is chosen for $f$ which is analytic in $\mathbb{C} \backslash[-1,1]$. Note that

$$
\arg (\sqrt{w})=\frac{1}{2} \arg w
$$


If $z \in \mathbb{C}$ and $\operatorname{Im} z>0$ then

$$
\arg (-(z \pm 1))=-\pi+\arg (z \pm 1)
$$

so

$$
\arg (\sqrt{-(z \pm 1)})=-\frac{\pi}{2}+\arg (\sqrt{z \pm 1}),
$$

and we have

$$
\sqrt{-(z \pm 1)}=-i \sqrt{z \pm 1}
$$

\section{Hence}

$$
f(-z)=\frac{-z}{i^{2} \sqrt{z+1} \sqrt{z-1}}=f(z) .
$$

Similarly if $\operatorname{Im} z<0$ then

$$
\sqrt{-(z \pm 1)}=i \sqrt{z \pm 1}
$$

and we have the same result.

Let $x>1$. Then

$$
\arg \sqrt{-(x \pm 1)}=\frac{\pi}{2},
$$

so

$$
f(-x)=\frac{-x}{\sqrt{-(x-1)} \sqrt{-(x+1)}}=\frac{x}{\sqrt{x+1} \sqrt{x-1}}=f(x) .
$$

Therefore $\left.f\right|_{\mathbb{C} \backslash[-1,1]}$ is even.

Let $\arg z \in(-\pi, \pi)$. For $\arg z \gtrless 0$,

$$
f(z)=\frac{\mp i z}{\sqrt{1+z} \sqrt{1-z}}
$$

since $z-1=e^{ \pm i \pi}(1-z)$. If $x \in(0,1)$, then

$$
f(x \pm i 0)=\frac{\mp i x}{\sqrt{1+x} \sqrt{1-x}}
$$

and

$$
f(-x \pm i 0)=\frac{ \pm i x}{\sqrt{1+x} \sqrt{1-x}}=-f(x \pm i 0) .
$$

Moreover, $f(0 \pm i 0)=0$. Therefore $\left.f\right|_{(-1,1) \pm i 0}$ maps to the imaginary axis and is an odd function of $x$.

If $x \in(0, \infty)$ then

$$
f(i x)=\frac{i x}{\sqrt{i x+1} \sqrt{i x-1}}=\frac{x}{\sqrt{1+x^{2}}},
$$

and

$$
f(-i x)=\frac{-i x}{\sqrt{-i x+1} \sqrt{-i x-1}}=\frac{x}{\sqrt{1+x^{2}}}
$$



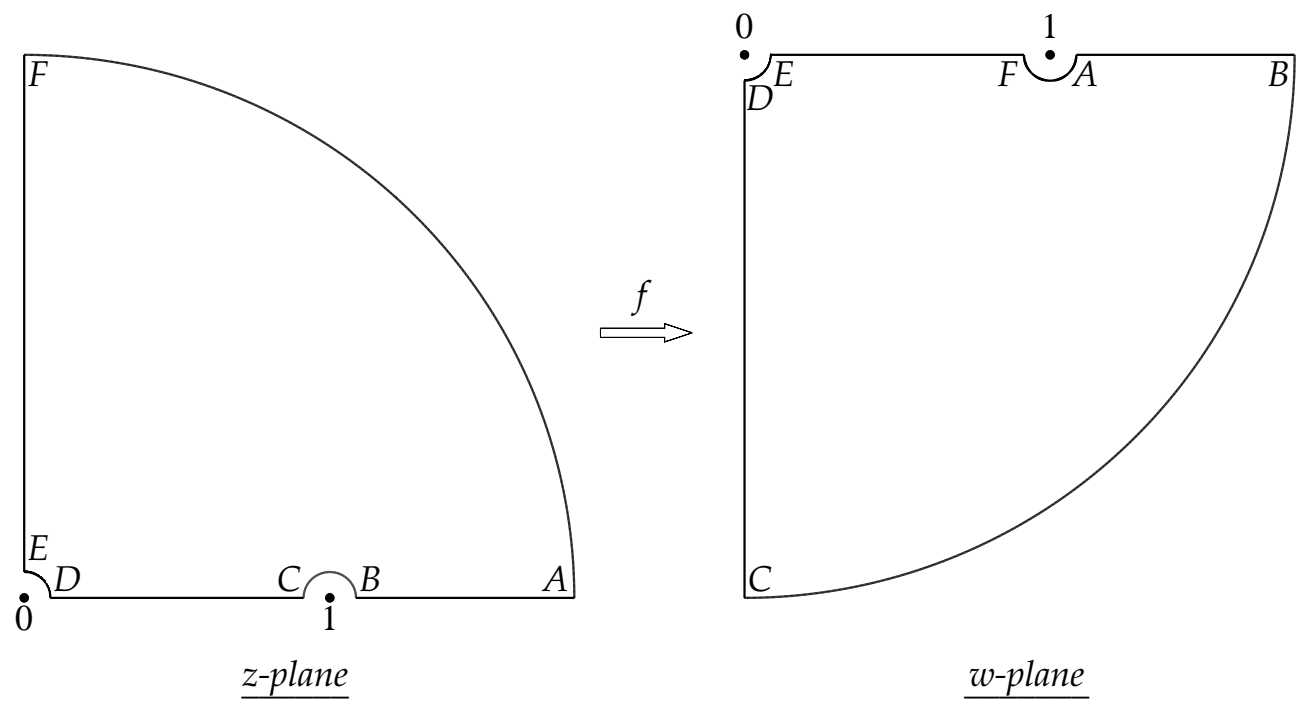

Figure 1. This figure shows how the function $f: \mathbb{C} \backslash\{-1,1\} \rightarrow \mathbb{C}$ defined by $f(z):=z /(\sqrt{z+1} \sqrt{z-1})$ conformally maps quadrant I into quadrant IV.

so $f$ maps both the positive and negative imaginary axes to the real interval $(0,1)$. Clearly $f(0)=0$. This completes the proof of $1,2,3$ and 4 .

Before we prove 5 we first show that $f$ maps quadrant I into quadrant IV. The derivative $f^{\prime}(z)=-(z+1)^{-3 / 2}(z-1)^{-3 / 2}$ is nowhere zero, and therefore $w=f(z)$ represents a conformal map of $\mathbb{C} \backslash\{-1,1\}$ (see for instance $[14, \S 1.9($ iv) $]$ ). Consider the closed contour represented in the $z$-plane of Fig. 1. In order to study the mapping properties of quadrant I by the map $f$, we look at the behavior of the map within and on a closed contour in quadrant I through a limiting process: Take the radii of the semi-circular segment $B C$ and the quarter-circular segment $D E$ tending towards zero, the radius of the quarter-circular segment $F A$ tending towards infinity, and match these segments to the straight line segments $A B, C D$, and $E F$ continuously.

The straight line segments are treated first, followed by the treatment of the circular segments. For $A B, z \in(1, \infty)$ and therefore $w(z)=1 / \sqrt{1-\left(1 / z^{2}\right)} \in(1, \infty)$. Therefore as $z \rightarrow A$, $w(z) \rightarrow 1+$ and as $z \rightarrow B, w(z) \rightarrow+\infty$. On $C D, z \in(0,1)$ so $w(z)=-i / \sqrt{\left(1 / z^{2}\right)-1} \in$ $-i(0, \infty)$. So as $z \rightarrow C, w(z) \rightarrow-i \infty$ and as $z \rightarrow D, w(z) \rightarrow-i 0$. On $E F, z \in i(0, \infty)$ thus $w(z)=z / \sqrt{1+z^{2}} \in(0,1)$ and as $z \rightarrow E, w(z) \rightarrow 0+$ and as $z \rightarrow F, w(z) \rightarrow 1-$. For the semi-circular segment $B C, z$ is near 1 and we write $z=1+\zeta$. Consider $\zeta=\epsilon e^{i \phi}$ with $\phi \in[0, \pi]$. Through the binomial expansion we can see that

$$
w(\zeta)=\frac{1}{\sqrt{2 \zeta}}+O(\sqrt{\zeta})=\frac{1}{\sqrt{2 \epsilon}} e^{-i \phi / 2}+O(\sqrt{\epsilon}) .
$$

Therefore as $z \rightarrow B, w(z) \rightarrow+\infty$ and as $z \rightarrow C, w(z) \rightarrow-i \infty$. For the quarter-circular segment $D E, z$ is near 0 and we write $z=\zeta=\epsilon e^{i \phi}$ with $\phi \in[0, \pi / 2]$. Through the binomial expansion we have

$$
w(\zeta)=-i \zeta+O\left(\zeta^{2}\right)=\epsilon e^{i(\phi-\pi / 2)}+O\left(\epsilon^{2}\right),
$$

and therefore as $z \rightarrow D, w(z) \rightarrow-i 0$ and as $z \rightarrow E, w(z) \rightarrow 0+$. For the quarter-circular segment $F A$, we write $z=\zeta$ and consider $\zeta=R e^{i \phi}$ with $\phi \in[0, \pi / 2]$ with $R$ chosen sufficiently large. Through the binomial expansion we have

$$
w(\zeta)=1+\frac{1}{2 \zeta^{2}}+O\left(\zeta^{-4}\right)=1+\frac{1}{2 R^{2}} e^{-2 i \phi}+O\left(R^{-4}\right),
$$

and therefore as $z \rightarrow F, w(z) \rightarrow 1-$. 
Hence the closed contour and its interior region in the $z$-plane are conformally mapped into the closed contour and its interior region in the $w$-plane shown in Fig. 1 . Through by the limiting process described above, we see that $f$ maps quadrant I into quadrant IV.

Due to the evenness of $f$, quadrants I \& III are mapped to quadrants IV, and quadrants II \& IV are mapped to quadrant I. Therefore if $z \in \mathbb{C} \backslash[-1,1]$ then $\operatorname{Re} f(z)>0$. This completes the proof of 5 .

Note. An anonymous referee has suggested an alternate proof that $\operatorname{Re} f(z)>0$ for all $\mathbb{C} \backslash[-1,1]$ using the minimum modulus principle.

Proof. Consider the function $g: \mathbb{C} \backslash\{-1,1\} \rightarrow \mathbb{C}$ defined by $g(z):=\exp f(z)$. A simple computation gives the modulus $|g(z)|=\exp \operatorname{Re} f(z)$. By (21), $|g(z)| \rightarrow e$ as $|z| \rightarrow \infty$. Let $x \in(-1,1)$ and consider $z=x+i \epsilon$ as $\epsilon \rightarrow 0^{ \pm}$, then

$$
f(z)=\frac{\mp i x}{\sqrt{1+x} \sqrt{1-x}}+O(\epsilon)
$$

so $|g(z)| \rightarrow 1$ in $(-1,1)$ (clearly $g$ is non-constant). In a small neighborhood $E$ of $z=1$, and by property 1 , in a small neighborhood $E^{\prime}$ of $z=-1$, we have through (20) that $|g(z)|>1$ in $E$ and $E^{\prime}$. The minimum modulus principle states (see for instance [16, p. 147]) that if $f$ is analytic, non-constant, and non-vanishing in an open connected subset $G$ of $\mathbb{C}$ then $|f(z)|$ cannot have a minimum in $G$. Let $G:=\mathbb{C} \backslash[-1,1]$, an open connected subset of $\mathbb{C}$. Since $f$ (and hence $g$ ) is analytic in $G$, and the exponential function is non-vanishing in $\mathbb{C}$, from the minimum modulus principle we have that $|g(z)|>1$ in $\mathbb{C} \backslash[-1,1]$ and therefore $\operatorname{Re} f(z)>0$ in $\mathbb{C} \backslash[-1,1]$. This completes the proof.

The range of $f$ is $\{z \in \mathbb{C}: \operatorname{Re} z \geq 0$ and $z \neq 1\}$. Every complex number in the range of the function is taken twice except for elements in $(0,1)$ and on the imaginary axis. These complex numbers are taken only once.

Consider the curve $f(z)$ when $\operatorname{Re} z=1$. In order to illustrate the behavior of this curve, take $z=1-i t$, where $t \in(0, \infty)$. By the above discussion, we know this line segment is mapped conformally from quadrant IV to quadrant I. This smooth curve asymptotically approaches the line $\operatorname{Re} w=\operatorname{Im} w$ from below and approaches the singularity at unity from the left side (i.e. from $\operatorname{Re} f(z)<1$ ). This can be seen through the asymptotics (cf. (20) and (21)), namely $\phi(\epsilon)=\pi-2 \sqrt{2 \epsilon}$ for the angle of approach to the singularity at unity as the distance $\epsilon \rightarrow 0$ and $\phi(R)=\pi / 4-11 \sqrt{2} /\left(64 R^{2}\right)$ (where terms up to fourth order have been included) as the radius $R$ tends towards infinity. If $f(1-i t)=x(t)+i y(t)$, then using elementary trigonometry one can show

$$
\begin{aligned}
& x(t)=\frac{1}{2 \sqrt{t\left(4+t^{2}\right)}}\left[(1+t) \sqrt{\sqrt{4+t^{2}}+2}+(t-1) \sqrt{\sqrt{4+t^{2}}-2}\right], \\
& y(t)=\frac{1}{2 \sqrt{t\left(4+t^{2}\right)}}\left[(1-t) \sqrt{\sqrt{4+t^{2}}+2}+(t+1) \sqrt{\sqrt{4+t^{2}}-2}\right],
\end{aligned}
$$

and therefore

$$
\begin{aligned}
& x^{2}(t)=\frac{1}{2 t\left(4+t^{2}\right)}\left[\left(1+t^{2}\right) \sqrt{4+t^{2}}+3 t+t^{3}\right], \\
& y^{2}(t)=\frac{1}{2 t\left(4+t^{2}\right)}\left[\left(1+t^{2}\right) \sqrt{4+t^{2}}-3 t-t^{3}\right] .
\end{aligned}
$$




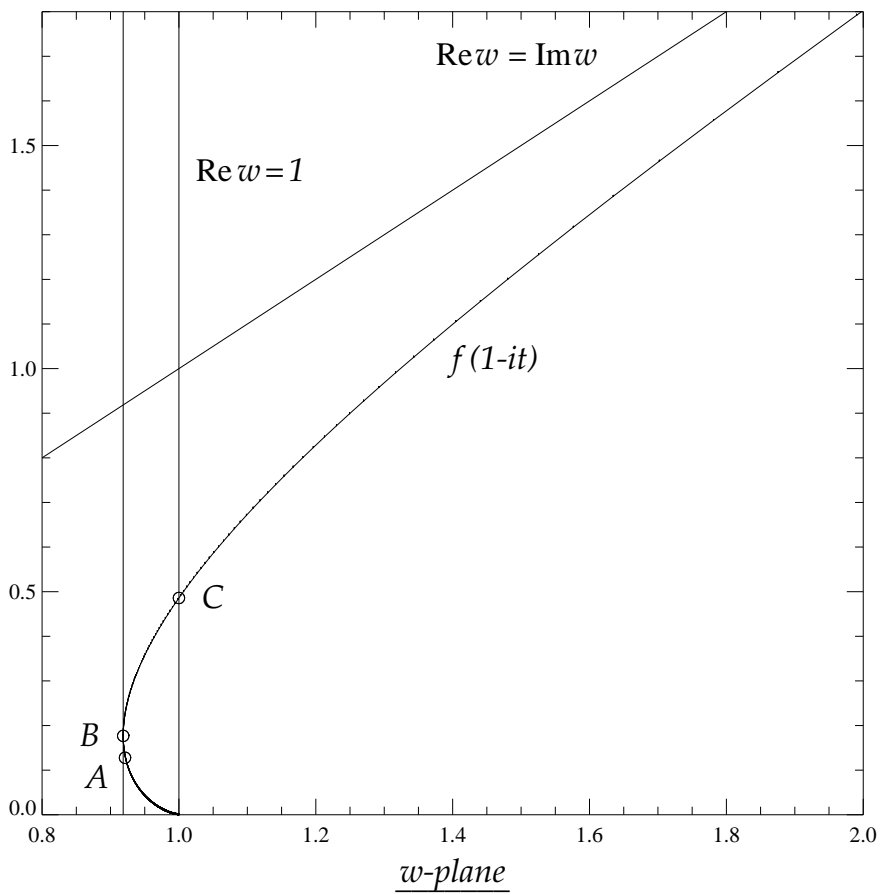

Figure 2. This figure shows the curve in the complex $w$-plane given by $f(1-i t)$ for $t \in(0, \infty)$. There are three lines indicated. One represents the line $\operatorname{Re} w=\operatorname{Im} w$. The other two represent the vertical lines passing through the points $B$ and $C$ respectively.

The distance squared from the origin is given by $\left(1+t^{2}\right) /\left(t \sqrt{4+t^{2}}\right)$, whose minimum occurs at $t=\sqrt{2}$ for

$$
(x, y)(\sqrt{2})=\left(\frac{1}{2} \sqrt{\frac{3 \sqrt{3}+5}{3}}, \frac{1}{2} \sqrt{\frac{3 \sqrt{3}-5}{3}}\right),
$$

portrayed by the point $A$ in Fig. 2. Due to the asymptotics of the curve near the singularity at unity, there exists a point at which the real part of this curve reaches a minimum value. By finding the minimum of $x^{2}(t)$, this point can be easily obtained and is shown in Fig. 2 by point $B$ and is given at $t=2 / \sqrt{3}$ by the point

$$
(x, y)\left(\frac{2}{\sqrt{3}}\right)=\left(\frac{3}{4} \sqrt{\frac{3}{2}}, \frac{1}{4 \sqrt{2}}\right) .
$$

Similarly one can find the point $C$ in Fig. 2 where $\operatorname{Re} f(z)=1$ to be given at $t=\sqrt{\sqrt{5}-2}$ at $y=t$.

\section{Acknowledgements}

I would like to thank Dr. A.F.M. ter Elst for extremely valuable discussions and acknowledge funding for time to write this paper from the Dean of the Faculty of Science at the University of Auckland in the form of a three month stipend to enhance University of Auckland 2012 PBRF Performance. I would like to express my gratitude to the anonymous referees whose helpful comments improved this paper. I would also like to thank F.W.J. Olver for helpful discussions. Part of this work was conducted while the author was a National Research Council Research Postdoctoral Associate in the Information Technology Laboratory of the National Institute of Standards and Technology. 


\section{References}

[1] Abramowitz M., Stegun I.A., Handbook of mathematical functions with formulas, graphs, and mathematical tables, National Bureau of Standards Applied Mathematics Series, Vol. 55, U.S. Government Printing Office, Washington, D.C., 1964.

[2] Apelblat A., Kravitsky N., Integral representations of derivatives and integrals with respect to the order of the Bessel functions $J_{\nu}(t), I_{\nu}(t)$, the Anger function $\mathbf{J}_{\nu}(t)$ and the integral Bessel function $\mathrm{Ji}_{\nu}(t), I M A J$. Appl. Math. 34 (1985), 187-210.

[3] Brychkov Yu.A., Handbook of special functions: derivatives, integrals, series and other formulas, CRC Press, Boca Raton, FL, 2008.

[4] Brychkov Yu.A., On the derivatives of the Legendre functions $P_{\nu}^{\mu}(z)$ and $Q_{\nu}^{\mu}(z)$ with respect to $\mu$ and $\nu$, Integral Transforms Spec. Funct. 21 (2010), 175-181.

[5] Cohl H.S., Derivatives with respect to the degree and order of associated Legendre functions for $|z|>1$ using modified Bessel functions, Integral Transforms Spec. Funct. 21 (2010), 581-588, arXiv:0911.5266.

[6] Cohl H.S., Rau A.R.P., Tohline J.E., Browne D.A., Cazes J.E., Barnes E.I., Useful alternative to the multipole expansion of $1 / r$ potentials, Phys. Rev. A 64 (2001), 052509, 5 pages.

[7] Cohl H.S., Tohline J.E., A compact cylindrical Green's function expansion for the solution of potential problems, Astrophys. J. 527 (1999), 86-101.

[8] Cohl H.S., Tohline J.E., Rau A.R.P., Srivastava H. M., Developments in determining the gravitational potential using toroidal functions, Astronom. Nachrichten 321 (2000), 363-372.

[9] Gradshteyn I.S., Ryzhik I.M., Table of integrals, series, and products, 7th ed., Elsevier/Academic Press, Amsterdam, 2007.

[10] Hobson E.W., The theory of spherical and ellipsoidal harmonics, Chelsea Publishing Company, New York, 1955.

[11] Lang S., Real and functional analysis, 3rd ed., Graduate Texts in Mathematics, Vol. 142, Springer-Verlag, New York, 1993.

[12] Magnus W., Oberhettinger F., Soni R.P., Formulas and theorems for the special functions of mathematical physics, 3rd ed., Die Grundlehren der mathematischen Wissenschaften, Band 52, Springer-Verlag, New York, 1966.

[13] Olver F.W.J., Asymptotics and special functions, AKP Classics, A K Peters Ltd., Wellesley, MA, 1997.

[14] Olver F.W.J., Lozier D.W., Boisvert R.F., Clark C.W. (Editors), NIST handbook of mathematical functions, U.S. Department of Commerce, National Institute of Standards and Technology, Washington, DC, 2010.

[15] Prudnikov A.P., Brychkov Yu.A., Marichev O.I., Integrals and series, Vol. 2, Special functions, 2nd ed., Gordon \& Breach Science Publishers, New York, 1988.

[16] Silverman R.A., Complex analysis with applications, Prentice-Hall Inc., Englewood Cliffs, N.J., 1974.

[17] Szmytkowski R., On the derivative of the Legendre function of the first kind with respect to its degree, J. Phys. A: Math. Gen. 39 (2006), 15147-15172, Addendum, J. Phys. A: Math. Theor. 40 (2007), 1488714891, Corrigendum, J. Phys. A: Math. Theor. 40 (2007), 7819-7820, arXiv:0907.3217.

[18] Szmytkowski R., A note on parameter derivatives of classical orthogonal polynomials, arXiv:0901.2639.

[19] Szmytkowski R., On parameter derivatives of the associated Legendre function of the first kind (with applications to the construction of the associated Legendre function of the second kind of integer degree and order), arXiv:0910.4550.

[20] Szmytkowski R., On the derivative of the associated Legendre function of the first kind of integer degree with respect to its order (with applications to the construction of the associated Legendre function of the second kind of integer degree and order), J. Math. Chem. 46 (2009), 231-260.

[21] Szmytkowski R., On the derivative of the associated Legendre function of the first kind of integer order with respect to its degree (with applications to the construction of the associated Legendre function of the second kind of integer degree and order), J. Math. Chem., to appear, arXiv:0907.3217.

[22] Szmytkowski R., Green's function for the wavized Maxwell fish-eye problem, J. Phys. A: Math. Theor. 44 (2011), 065203, 14 pages.

[23] Watson G.N., A treatise on the theory of Bessel functions, 2nd ed., Cambridge Mathematical Library, Cambridge University Press, Cambridge, 1944.

[24] Whipple F.J.W., A symmetrical relation between Legendre's functions with parameters $\cosh \alpha$ and $\operatorname{coth} \alpha$, Proc. London Math. Soc. 16 (1917), 301-314. 